Published in final edited form as:

J Perinatol. 2009 March ; 29(3): . doi:10.1038/jp.2008.192.

\title{
Birth asphyxia survivors in a developing country
}

\author{
DR Halloran ${ }^{1}$, E McClure ${ }^{2}$, H Chakraborty ${ }^{2}$, E Chomba ${ }^{3}$, LL Wright ${ }^{4}$, and WA Carlo ${ }^{5}$ \\ ${ }^{1}$ Department of Pediatrics, Saint Louis University, Saint Louis, MI, USA \\ ${ }^{2}$ Department of Statistics and Epidemiology, RTI International, RTP, NC, USA \\ ${ }^{3}$ University Teaching Hospital, Lusaka, Zambia \\ ${ }^{4}$ Center for Research for Mothers and Children, National Institute of Child Health and Human \\ Development, Bethesda, MD, USA \\ ${ }^{5}$ Department of Pediatrics, University of Alabama at Birmingham, Birmingham, AL, USA
}

\begin{abstract}
Objective-Determine the baseline incidence of birth asphyxia in neonatal intensive care unit (NICU) survivors in a developing country and the early neurodevelopmental outcomes of such infants.
\end{abstract}

Study Design-This cross-sectional, prospective study collected diagnostic and examination findings on all infants seen in the University of Zambia NICU follow-up clinic over a 4-week period.

Result-Of the 182 infants, 42 (23\%) had a clinical diagnosis of birth asphyxia. Of 42 infants with birth asphyxia, $13(31 \%)$ had an abnormal neurologic examination during the clinic visit; in contrast, 13 of 141 infants without birth asphyxia (9\%) had an abnormal examination (odds ratio 4.4, $95 \%$ confidence interval: $1.8,10.4$ ).

Conclusion-Birth asphyxia survivors account for almost a quarter of NICU survivors in a developing country and half of those with an abnormal neurologic examination. Studies are necessary to determine the percent of birth asphyxia survivors who have permanent motor and cognitive disabilities.

\section{Keywords}

asphyxia neonatorum; child development; neurologic manifestations; developing countries; Zambia; infant

\section{Introduction}

Globally, $23 \%$ of neonatal deaths ${ }^{1}$ and $10 \%$ of all deaths in children less than 5 years of age $^{2,3}$ are estimated to occur as a result of birth asphyxia, defined as failure to initiate and sustain normal breathing at birth, ${ }^{4}$ and account for one million deaths each year worldwide. A multicenter study involving hospitals in eight African countries including Zambia found birth asphyxia (defined as an Apgar score $<7$ at $5 \mathrm{~min}$ ) to be the leading (23\%) cause of

(C) 2008 Nature Publishing Group All rights reserved.

Correspondence: Dr DR Halloran, Department of Pediatrics, Saint Louis University, 1465 South Grand Blvd., St. Louis, MO 63104, USA.dhallor2@slu.edu.

Conflicts of interest/Disclosure

The authors have no conflicts of interest to report. 
neonatal mortality. ${ }^{4}$ In spite of the large impact that birth asphyxia had on neonatal mortality, this study found that $87 \%$ of infants diagnosed with birth asphyxia survived to discharge home.

Survivors of birth asphyxia are at risk for neurodevelopmental sequelae including motor and cognitive disabilities. ${ }^{5-9}$ One of the best population studies of asphyxia in a developing country reported that $18 \%$ of survivors of mild to moderate birth asphyxia had neonatal encephalopathy and permanent severe neurologic impairment. ${ }^{10}$ Birth asphyxia was a common cause of mental retardation, cerebral palsy and other neurodevelopmental disorders.

Evaluation for long-term sequelae can best be performed when a child reaches an age at which established screening tools can be utilized, generally at least 18 months; ${ }^{11-13}$ however, many babies may have evidence of disabilities in early infancy. Therefore, neurodevelopmental examinations as early as 6 weeks of age may provide an indication of the magnitude of the problem. ${ }^{10}$

Although accurate estimates of neurodevelopmental sequelae from birth asphyxia in developing countries are not available, studies in developed countries have found that 15 to $18 \%$ of infants who suffer moderate to severe asphyxia are disabled by 8 years of age. ${ }^{7}$

Moderate to severe asphyxia was defined as altered consciousness with altered muscle tone or primitive reflexes after $1 \mathrm{~h}$ of age along with intrapartum fetal distress, immediate neonatal distress, or immediate neonatal resuscitation. Given current sociocultural factors in developing countries such as Zambia, the burden of care is likely to be much greater than in developed countries. ${ }^{14}$ Poverty, illiteracy, low status of women, poor hygiene, lack of clean water and sanitation, family inability to recognize danger signs, and inadequate access to medical care all increase the risk for morbidity following birth asphyxia in developing countries. Often rehabilitation resources are not available in these settings.

Birth asphyxia is likely a leading cause of neonatal death in most developing countries such as Zambia; ${ }^{15,16}$ however, prospective studies to assess the early morbidity following birth asphyxia have not been performed in Sub-Saharan Africa. Although prevention and prompt treatment of birth asphyxia and reduction of its sequelae are among the developing world's top health care priorities, ${ }^{17-19}$ studies are needed to determine the frequency of such sequelae and the resulting burden on the health system and society. This study was designed to determine the baseline incidence of birth asphyxia in neonatal intensive care unit (NICU) survivors and the early neurodevelopmental outcomes of these infants.

\section{Methods}

\section{Setting}

The study was conducted in Lusaka, the capital of Zambia, a developing country in SubSaharan Africa. Health indicators in Zambia are poor with actual per capita public expenditure on health at $\$ 11$ per year and total expenditure on health at $6.6 \%$ of the gross domestic product. ${ }^{20}$ Life expectancy is 39 years, due in large part to economic deterioration and the HIV/AIDS epidemic. ${ }^{21,22}$ Infant mortality is estimated to be 95 per 1000 live births, and the under age 5 years mortality rate is 182 per $1000 .^{21,23}$

The public health system serves over $95 \%$ of neonates in its catchment area and is divided into 25 primary health care centers, including 11 delivery centers and one referral hospital. High-risk patients are routinely referred prenatally, during labor, or after birth to the University Teaching Hospital (UTH), the only teaching hospital which is affiliated with the University of Zambia. The NICU has three ventilators, 34 incubators and 38 cots. The unit, 
which has approximately 1500 deliveries per year, is staffed by three to five nurses per shift and a total of four to six physicians (a senior consultant, one or two registrars and two or three junior staff). The NICU mortality rate is $41 \% ; 60$ to $65 \%$ of deaths occur in the first 3 days following admission and 75 to $85 \%$ by the end of the first week (E Chomba, personal communication, February 27, 2006).

The infants cared for in the UTH NICU include all infants who survive following severe birth asphyxia and other significant delivery complications in the Lusaka area (over 40000 deliveries per year) and are referred to the NICU follow-up clinic 4 to 6 weeks after discharge. This clinic only follows infants cared for in the UTH NICU. Standard follow-up in the NICU follow-up clinic is every 4 weeks but may be altered for clinical indications. Patients are discharged from the NICU follow-up clinic to the local health clinic when routine care is required or to the UTH neurology subspecialty clinic when there is persistent evidence of delayed neurodevelopment beyond 3 months of age.

\section{Study design}

A cross-sectional, prospective clinic data collection and retrospective hospital chart review was completed on all infants who presented to the NICU follow-up clinic for a 4-week period between March and April 2005. The percent of infants who return for follow-up is not routinely tracked but is estimated to be 75 to $85 \%$ based on hospital records.

A structured data sheet was used to abstract information from the chart for each infant seen in the clinic during the study period. Infant demographics, including date of birth, birth weight and gender, were collected. Delivery data included maternal parity, location (home, clinic or hospital) and method of delivery (vaginal or cesarean section), and Apgar score (1, 5 , and $10 \mathrm{~min}$ ) when available.

All diagnoses documented during the hospital course were recorded; therefore, infants may have had multiple diagnoses. Only those infants who received phototherapy were assigned a diagnosis of neonatal jaundice.

Birth asphyxia was a clinical diagnosis made during initial assessment by the UTH physician, based on delivery history and/or Apgar score using the WHO (World Health Organization) definition of failure to initiate and sustain normal breathing at birth. ${ }^{24}$ For this study the definition of clinical asphyxia was defined by hospital protocol as infants with a 5min Apgar score <7; however, the clinical diagnosis may have been made in the absence of Apgar scores for home deliveries. This was similarly applied to both term and preterm infants. Birth asphyxia was dichotomized as present or absent without assessment of severity. The rate of clinical diagnosis of birth asphyxia in this population was compared to rates of birth asphyxia calculated with previously published criteria including the following: Bao criteria ${ }^{25}$ (5-min Apgar score <6), Ellis criteria ${ }^{10}$ (5-min Apgar score $₫ 4$ ) and Volpe criteria $^{26}$ (5-min Apgar score <4).

Information recorded at the clinic follow-up visit included date of visit, weight, skin color (normal or pale), neurologic examination, problem list and plan of care. Neurologic examinations were performed by three senior staff physicians, masked to clinical course, during the follow-up visit. The physician was aware of and considered the infant's age and level of prematurity when assessing tone. The neurologic examinations were dichotomized as normal or abnormal. An abnormal exam included any exam with increased or decreased head control and/or increased, decreased or mixed overall tone. Other examination findings, including any concerns with respect to hearing or vision impairment, were recorded as openended text. The plan of care was abstracted including when and where the infant was scheduled for follow-up (NICU follow-up clinic, neurology clinic or 'under five' clinic for 
routine care), what medications were prescribed, and whether or not breastfeeding counseling was ordered. Other follow-up was recorded in an open-ended fashion. At the time the study was conducted, no form of early intervention for infant stimulation was present in the community. No information was obtained for those infants who were not seen in the NICU follow-up clinic.

A few infants were seen more than once during the period of chart abstraction. In these cases, the last follow-up visit for each of these infants was included in the analyses.

\section{Data analysis}

Odds ratios and confidence intervals (CIs) were calculated for the entire population as well as for those infants born at (inborn) or outside (outborn—clinic or home) the hospital. Data analyses included descriptive statistics, $X^{2}$ and Fisher's exact tests, and multivariable backward regression analysis using SAS systems (SAS Institute Inc., Cary, NC, USA).

The study was approved by the Institutional Review Board at the University of Alabama at Birmingham, the Research Triangle Institute, as well as the Research Ethics Committee at the UTH in Lusaka, Zambia.

\section{Results}

A total of 211 clinic visits were prospectively abstracted. A total of 19 infants were seen twice and 5 infants were seen three times during the study period. A total of 182 infants remained for analyses. The mean age at the time of the visit was 67 days (s.d. \pm 58 days) with a median of 50 days.

\section{History}

There was a slight predominance of males in the study population $(99,55 \%)$. The majority of infants $(140,79 \%)$ were born at UTH versus a clinic $(21,12 \%)$ or at home $(17,9 \%)$. Vaginal delivery was the most common method of delivery $(137,78 \%)$. Maternal parity of 1 to 2 was most common $(114,65 \%)$ versus parity $\geq 3(48,27 \%)$ or nulliparity $(14,8 \%)$. The mean birth weight was $2243 \mathrm{~g}$ (s.d. $\pm 781 \mathrm{~g}$ ); the median was $2000 \mathrm{~g}$. Suspected sepsis was the leading clinical diagnosis (74\%) made during the initial NICU stay among inborn and outborn survivors (Tables 1 and 2). A total of 11 infants (6\%) had a positive blood culture (Klebsiella (5), Proteus (2), Staphylococcus (1), Enterococcus (1), Enterobacter (1), Streptococcus (1) and Salmonella (1)).

A 1-min Apgar score was documented for 140 of 182 infants (77\%); 114 infants (63\%) had a 5-min and 34 infants (19\%) had a 10-min Apgar score documented. A total of 42 infants (23\%) had a clinical diagnosis of birth asphyxia recorded on the hospital chart. Six of these infants did not have a 5-min Apgar score. Of the 36 infants, 31 (86\%) with Apgar scores met Bao criteria ${ }^{24}$ for birth asphyxia (Apgar score $<7$ ). Eight $(22 \%)$ infants had birth asphyxia based on Ellis criteria ${ }^{10}$ (Apgar score $\$ 4$ ) whereas one infant had birth asphyxia based on Volpe criteria26 (Apgar score <4).

A total of 30\% (35) of infants born in the hospital had a clinical diagnosis of birth asphyxia versus 20\% (4) born in the clinic and $12 \%$ (2) born at home. A total of 22\% (30) of infants born by vaginal delivery had birth asphyxia versus $26 \%$ (10) born by cesarean section. A total of $24 \%$ (20) of female and $21 \%$ (21) of male infants had birth asphyxia. A total of 2 (14\%) infants born to nulliparous mothers, 33 (29\%) infants with maternal parity of 1 to 2 and $4(8 \%)$ infants with maternal parity $\geq 3$ had birth asphyxia. These factors were not predictive of birth asphyxia. 
Infants with birth weight $<1500 \mathrm{~g}$ were $12.6(95 \% \mathrm{CI} 2.8,57.2)$ times more likely to have birth asphyxia than infants $>2500 \mathrm{~g}$ whereas infants 1500 to $2500 \mathrm{~g}$ were 24.6 (95\% CI 7.1, 85.3) times more likely to have birth asphyxia. This pattern persisted in inborn but not outborn infants (Tables 1 and 2). Infants with a history of seizures (OR 7.4 (95\% CI 2.0, 29.5)) and multiple births (OR 24.5 (95\% CI 1.5, 409)) were more likely to have a clinical diagnosis of birth asphyxia on bivariate analysis whereas infants with respiratory distress were less likely to have birth asphyxia (OR $0.1(95 \%$ CI $0.02,0.3)$ ). Most of these relationships persisted in the inborn (Table 1) but not the outborn infants (Table 2). Multivariable regression analysis was not possible due to multicolinearity and small sample size.

\section{Physical findings}

A total of 26 infants (14\%) had an abnormal neurologic examination. Infants 1500 to $2500 \mathrm{~g}$ were $3.8(95 \%$ CI $1.3,11.1)$ more likely to have abnormal neurologic examinations than infants $>2500 \mathrm{~g}$. This relationship did not persist in the subanalyses (Tables 1 and 2). A total of 22 infants (16\%) born in the hospital had an abnormal neurologic exam versus $2(10 \%)$ born in the clinic and $1(6 \%)$ born at home. A total of 19 infants (14\%) born by vaginal delivery had an abnormal exam versus $5(13 \%)$ by cesarean delivery. A total of $12 \%$ (10) of female and 16\% (16) of male infants had an abnormal neurologic exam. A total of 1 (7\%) infant born to nulliparous mothers, $18(16 \%)$ infants with maternal parity of 1 to 2 and 5 (10\%) infants with maternal parity $\geq 3$ had an abnormal exam. These factors were not predictive of an abnormal neurologic examination.

Infants with clinical birth asphyxia were 4.4 times more likely to have an abnormal neurologic examination than nonasphyxiated infants (95\% CI: 1.8, 10.4). These findings persisted in the inborn analysis only (Tables 1 and 2). A total of 50\% (13 infants) of the 26 infants with an abnormal neurologic examination had clinical birth asphyxia (Figure 1). Of the 26, 10 infants with an abnormal neurologic examination met $\mathrm{Bao}^{24}$ criteria for birth asphyxia whereas only 1 infant with an abnormal neurologic examination met Ellis ${ }^{10}$ criteria for birth asphyxia; none met Volpe ${ }^{26}$ criteria for birth asphyxia. On the basis of backward selection regression analysis, clinical birth asphyxia was the only statistically significant predictor of an abnormal neurologic examination.

\section{Treatment plan}

Of the 26, 22 (85\%) infants with an abnormal neurologic exam were scheduled for continued observation in the NICU follow-up clinic; the remaining 4 infants (15\%) were referred for continued follow-up to the pediatric neurology clinic for developmental delay. A total of 53 infants (29\%) seen in the clinic were referred for routine care to the 'under five' regional clinics with normal neurologic exams; the remaining 103 infants (57\%) were scheduled to return to the NICU follow-up clinic for continued monitoring. Of the 42 infants with a diagnosis of birth asphyxia, 2 infants (5\%) were referred to neurology clinic, 7 infants $(17 \%)$ were referred to regional clinics for routine care and 33 infants (79\%) were scheduled to return to the NICU follow-up clinic.

Although the majority $(128,71 \%)$ of infants in the study were scheduled for follow-up in 4 weeks, 5 (3\%) were scheduled for follow-up in 3 weeks, 17 (9\%) at 2 weeks and 19 (11\%) at 1 week. The remaining 12 infants were scheduled for follow-up in 6 to 8 weeks. Additional specialty follow-up was scheduled for maternal HIV testing (3), breastfeeding counseling (32) and physical therapy (3). In addition, two children were referred to general surgery for inguinal hernias, one child was referred to general surgery for an umbilical hernia, one child was referred to orthopedics for bilateral clubfoot, one child was referred to plastic surgery for webbing of the fingers and toes, and one child was referred to plastic 
surgery for a cleft of the right cheek with lip and mandible deformities. Although breastfeeding was nearly universal in the population, 32 children were referred for additional breastfeeding counseling and support. Six children were readmitted for further evaluation and monitoring with a diagnosis of possible sepsis (4), jaundice (1) and pneumonia (1).

\section{Discussion}

Birth asphyxia survivors account for $23 \%$ of infants seen in a NICU follow-up clinic in a developing country. In this study, assessment of early neurodevelopmental outcomes revealed that $31 \%$ of surviving infants with clinical birth asphyxia had abnormal neurologic examinations at early follow-up. Half of the children seen in the NICU follow-up clinic with abnormal neurologic examinations had birth asphyxia. To the best of our knowledge this has not been shown before.

Although birth asphyxia is known to be a leading cause of neonatal mortality, survivors of birth asphyxia may suffer from hypoxic-ischemic brain injury affecting vulnerable areas of the brain leading to neurodevelopmental sequelae including problems with sensory-motor, auditory and language processing. ${ }^{6}$ Kinoti, ${ }^{4}$ in a multicenter study in Sub-Saharan Africa, found that $87 \%$ of infants with birth asphyxia, using the WHO definition, survived to discharge; however, he did not assess the neurologic outcome of these survivors. We assessed the early neurologic examinations of all birth asphyxia survivors who presented for follow-up and found birth asphyxia to be the leading diagnosis associated with an abnormal exam in this population of NICU survivors.

Our findings are consistent with those of Ellis et al., ${ }^{10}$ who found that $20 \%$ of infants with neonatal encephalopathy had neurologic impairments at 1 year follow-up versus $50 \%$ in our study, though at an earlier age. The Ellis study was conducted at a single hospital in Nepal, and serial follow-up was conducted at 6 weeks, 6 months and 1 year. At 1 year, 22\% of infants had been lost to follow-up including 3 of 7 infants who had grade 2 neonatal encephalopathy with impairment at 6 weeks of age which, in addition to the extended length of follow-up in the study, may explain the discrepancy in the rate of abnormal neurologic exams between the studies.

There is not full agreement on the research definition for birth asphyxia in the developing world. As described above, criteria by Bao, Volpe and Ellis have been developed based on Apgar scores. To allow comparison with previously published work on birth asphyxia in developing countries, we determined the rate of birth asphyxia in our population using these criteria. A total of $86 \%$ of our cohort of infants with a clinical diagnosis of birth asphyxia and an Apgar score met Bao's criteria for birth asphyxia leading to conclusions similar to ours about the impact of birth asphyxia on neurodevelopmental delay. However, future studies which utilize the strict definition of birth asphyxia offered by Volpe and/or Ellis may, in fact, miss children who suffer from birth asphyxia and the resulting neurologic sequelae. Infants born without assignment of an Apgar score, whether at home or in a clinic, are at risk for birth asphyxia and should be included in studies to identify birth asphyxia morbidity, particularly in light of previous studies which have questioned the validity of the Apgar score as a predictor of birth asphyxia. ${ }^{27}$ Although the World Federation of Neurology Group defined birth asphyxia as 'a condition of impaired gas exchange leading ... to progressive hypoxemia and hypercapnia,' obtaining a blood gas in developing countries for each infant who presents with a concerning history is impossible. ${ }^{28}$

This study utilized a clinical diagnosis based on the WHO definition of birth asphyxia as failure to initiate and sustain breathing at birth, the only feasible definition in this setting. Given the small sample size for certain outcomes, for example birth asphyxia in infants born 
at home, we are unable to make definitive conclusions with respect to risk for these outcomes. Neurologic examinations were performed at the time of the visit, given the average age of 67 days at the time of the visit, neurologic findings may be unreliable and may improve with time. However, data from developed countries indicate that early intervention is critical to improved motor and cognitive impairments and such interventions are currently unavailable in Zambia. ${ }^{29}$

All infants who presented for follow-up were included in the analyses; however, the characteristics of the infants who failed to keep the appointment are not known. Although previous studies in developed countries have found a bias in the outcomes of infants who present for long-term follow-up, ${ }^{30-32}$ it is unclear if this is true in developing countries. Transportation is difficult to afford for many families, regardless of the health of the infant, and likely contributed significantly to the lack of follow-up for certain infants. Ultimately, this study examined the morbidity associated with survivors of birth asphyxia who attended the follow-up; our findings, therefore, represent only a portion of the true burden of birth asphyxia. Although subanalyses for inborn and outborn infants were presented, the number of infants born at home or in the clinics was small limiting conclusions about the health of infants born outside the hospital.

The study was conducted in a single center which is the referral center for all the high-risk deliveries and newborn infants in Lusaka. As described in the 'Methods' section, we anticipate the patient population is representative of other large municipalities in SubSaharan Africa because of its similarities in demography, life expectancy and infant mortality. ${ }^{20,21}$ Formal neurological testing was not performed during the initial hospitalization; however, later neurologic examinations can be considered reliable and provide an early assessment of infants when performed by experienced physicians. ${ }^{6}$ In future studies, long-term sequelae will be measured using established screening tools.

\section{Conclusions}

Birth asphyxia survivors accounted for $23 \%$ of infants seen in a NICU follow-up clinic in a developing country and $50 \%$ of infants with early, abnormal neurologic examinations. Further research to determine the long-term developmental consequences of birth asphyxia is needed. Birth asphyxia survivors are likely to be a large contributor to early deaths and long-term disability in developing countries. Cost-effective strategies to reduce the high burden of birth asphyxia are urgently required.

\section{Acknowledgments}

We thank all of the patients, physicians, nurses and medical records personnel at The University Teaching Hospital in Lusaka, Zambia, who helped make this study a success. In addition, thank you to Carolyn S Ashworth, MD, $\mathrm{MPH}$ at the University of Alabama at Birmingham whose understanding and flexibility made the work in Zambia possible.

This study was supported by Global Network for Women's and Children's Health Research (HD 434646-03) National Institute of Health, The Bill and Melinda Gates Foundation; BRAIN Planning Grant for the Developing World (TW006703-02) — National Institute of Child Health and Development; UAB Perinatal Health and Human Development Pilot Grant; KL2 RR024994-ICTS Multidisciplinary Clinical Research Career Development Program.

\section{References}

1. Lawn JE, Cousens S, Zupan J. Lancet Neonatal Survival Steering Team. 4 million neonatal deaths: when? Where? Why? Lancet. 2005; 365:891-900. [PubMed: 15752534] 
2. Jones G, Steketee RW, Black RE, Zulfigar AB, Morris SS. Bellagio Child Survival Study Group. How many child deaths can we prevent this year? Lancet. 2003; 362:65-71. [PubMed: 12853204]

3. Bryce J, Boschi-Pinto C, Shibuya K, Black RE. WHO Child Health Epidemiology Reference Group. WHO estimates of the causes of death in children. Lancet. 2005; 365:1147-1152. [PubMed: 15794969]

4. Kinoti SS. Asphyxia of the newborn in East, Central, and Southern Africa. East Af Med J. 1993; 70:422-433.

5. Simon NP. Long-term neurodevelopmental outcome of asphyxiated newborns. Resuscitation Fetus Newborn. 1999; 26:767-778.

6. Robertson CMT, Finer NN. Long-term follow-up of term neonates with perinatal asphyxia. Clin Perinatol. 1993; 20:483-499. [PubMed: 7689432]

7. Robertson CMT, Finer NN, Grace MGA. School performance of survivors of neonatal encephalopathy associated with birth asphyxia at term. J Pediatr. 1989; 114:753-760. [PubMed: 2469789]

8. Cioni G, Prechtl HFR, Ferrari F, Paolicelli PB, Einspieler C, Roversi MF. Which better predicts later outcome in fullterm infants: quality of general movements or neurological examination? Early Hum Dev. 1997; 50:71-85. [PubMed: 9467695]

9. Brookhouser PE. Sensorineural hearing loss in children. Pediatr Clin North Am. 1996; 43(6):11951216. [PubMed: 8973508]

10. Ellis M, Manandhar N, Shrestha PS, Shrestha L, Manadhar DS, de L Costello AM. Outcome at 1 year of neonatal encephalopathy in Kathmandu, Nepal. Dev Med Child Neurol. 1999; 41:689-695. [PubMed: 10587046]

11. Durkin MS, Wang W, Shrout PE, Zaman SS, Hasan ZM, Desai P, et al. Evaluating a ten question screen for childhood disability: reliability and internal structure in different cultures. J Clin Epidemiol. 1995; 48:657-666. [PubMed: 7537327]

12. Palisano R, Rosenbaum P, Walter S, Russell D, Wood E, Galuppi B. Development and reliability of a system to classify gross motor function in children with cerebral palsy. Dev Med Child Neurol. 1997; 39:214-223. [PubMed: 9183258]

13. Chopra G, Verma IC, Seetharaman P. Development and assessment of a screening test for detecting childhood disabilities. Indian J Pediatr. 1999; 66:331-335. [PubMed: 10798079]

14. Stoll BJ, Measham AR. Children can't wait: improving the future for the world's poorest infants. J Pediatr. 2001; 139:729-733. [PubMed: 11713454]

15. Lawn, JE.; Cousens, SN.; Wilczyneska, K. Estimating the Causes of Four Million Neonatal Deaths in the Year 2000; Statistical Annex-The World Health Report 2005. Geneva: World Health Organization; 2005.

16. English M, Muhoro A, Aluda M, Were S, Ross A, Peshu N. Outcome of delivery and causespecific mortality and severe morbidity in early infancy: a Kenyan district hospital birth cohort. Am J Trop Med Hyg. 2003; 69:228-232. [PubMed: 13677381]

17. Sall M, Sylla J. African Prime Ministers take lead in child survival. Lancet. 2005; 366:198-199.

18. Martines J, Paul VK, Bhutta ZA, Koblinsky M, Soucat A, Walker N, et al. Neonatal survival: a call for action. Lancet. 2005; 365:1189-1197. [PubMed: 15794974]

19. Lawn JE, Manandhar A, Haws RA, Dramstadt GL. Reducing one million child deaths from birth asphyxia—a survey of health systems gaps and priorities. Health Res Policy Syst. 2007; 5:4. [PubMed: 17506872]

20. The World Health Report 2005-Make Every Mother and Child Count. Geneva: World Health Organization; 2005. p. 200-204.Accessed at http://www.who.int/whr/2005/en/ [on November 18, 2008]

21. The World Health Report 2005-Make Every Mother and Child Count. Geneva: World Health Organization; 2005. p. 182-186.Accessed at http://www.who.int/whr/2005/en/ [on November 18, 2008]

22. Mathers, Cd; Sadana, R.; Salomon, JA.; Murray, CJL.; Lopez, AD. Healthy life expectancy in 191 countries, 1999. Lancet. 2001; 357:1685-1691. [PubMed: 11425392]

23. UNICEF. Information by country. Issues facing children in Zambia. Accessed at http:// www.unicef.org/infobycountry/zambia_1391.html. 
24. Maternal and Newborn Health/Safe Motherhood Unit. Basic Newborn Resuscitation: A Practical Guide. Geneva: World Health Organization; 1997. Division of Reproductive Health (Technical Support); p. 4Accessed at http://www.who.int/reproductive-health/publications/ newborn_resuscitation/basic_newborn_resuscitation.pdf [on February 27, 2008]

25. Bao XL, Yu RJ, Li ZS. 20-item neonatal behavioral neurological assessment used in predicting prognosis of asphyxiated newborn. Chin Med J. 1993; 106:211-215. [PubMed: 8325146]

26. Volpe, J. Hypoxic-ischemic encephalopathy: clinical aspects. In: Volpe, J., editor. Neurology of the Newborn. 3rd edn.. Philadelphia: WB Saunders; 1995. p. 279-344.

27. American Academy of Pediatrics, American College of Obstetricians and Gynecologistis. Use and abuse of the Apgar score. Pediatrics. 1996; 98:141-142. [PubMed: 8668389]

28. Bax M, Nelson KB. Birth asphyxia: a statement. World Federation Neurology Group. Dev Med Child Neurol. 1993; 35:1022-1024. [PubMed: 8224557]

29. Spittle AJ, Orton J, Doyle LW, Boyd R. Early developmental intervention programs post hospital discharge to prevent motor and cognitive impairments in preterm infants. Cochrane Database Syst Rev. 2007; 2:CD005495. [PubMed: 17443595]

30. Tin W, Fritz S, Wariyar W, Hey E. Outcome of very preterm birth: children reviewed with ease at 2 years differ from those followed up with difficulty. Arch Dis Child Fetal Neonatal Ed. 1998;

79:F83-F87. [PubMed: 9828731]

31. Castro L, Yolton K, Haberman B, Roberto N, Hansen NI, Ambalavanan N, et al. Bias in reported neurodevelopmental outcomes among extremely low birth weight survivors. Pediatrics. 2004; 114:404-410. [PubMed: 15286223]

32. Callanan C, Doyle L, Rickards A, Kelly E, Ford G, Davis N. Children followed with difficulty: how do they differ? J Paediatr Child Health. 2001; 37:152-156. [PubMed: 11328470] 


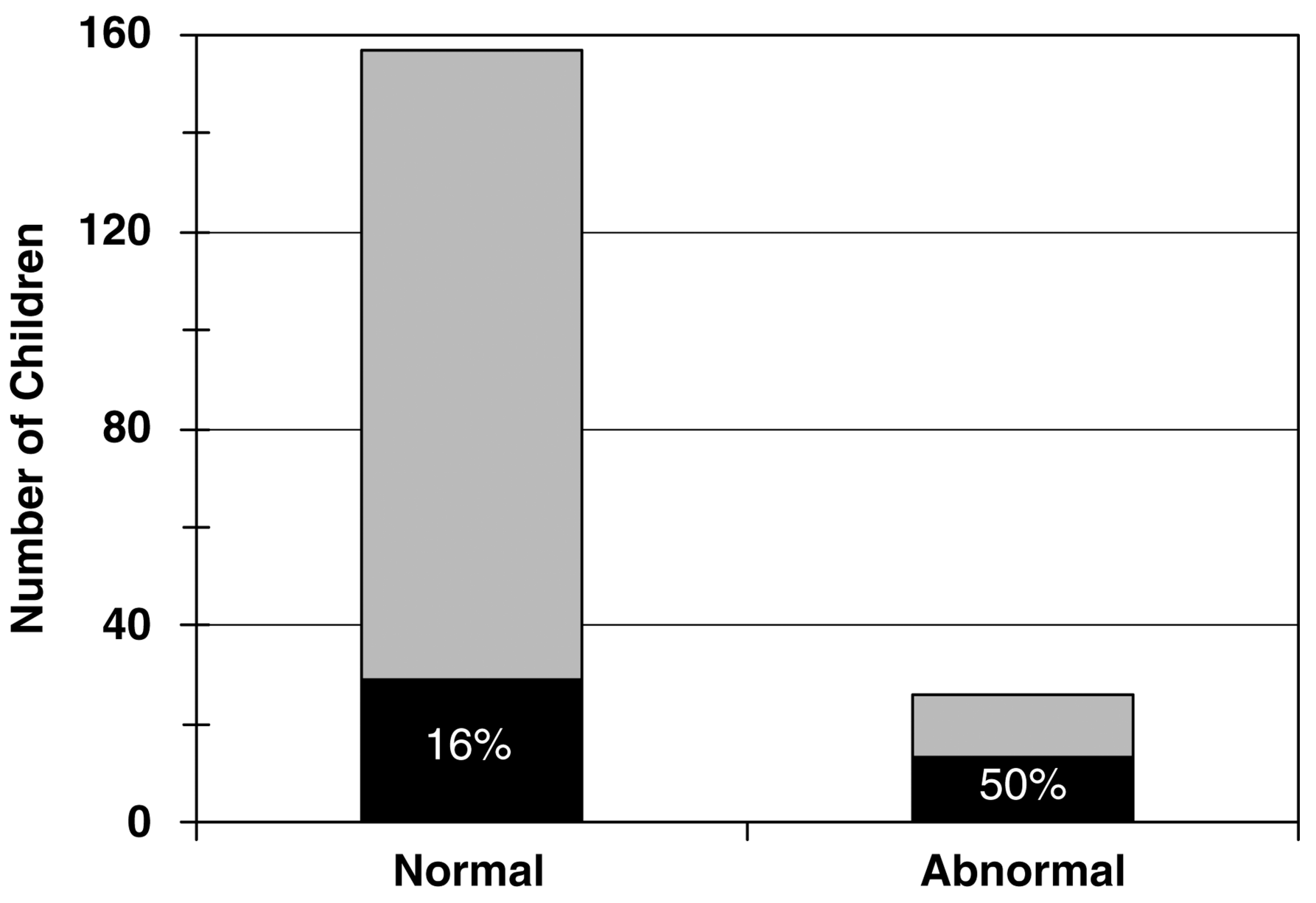

\section{Neurologic Examination $\square$ Birth Asphyxia $\square$ No Birth Asphyxia}

Figure 1.

Neurologic examination findings for infants with and without a clinical diagnosis of birth asphyxia. 


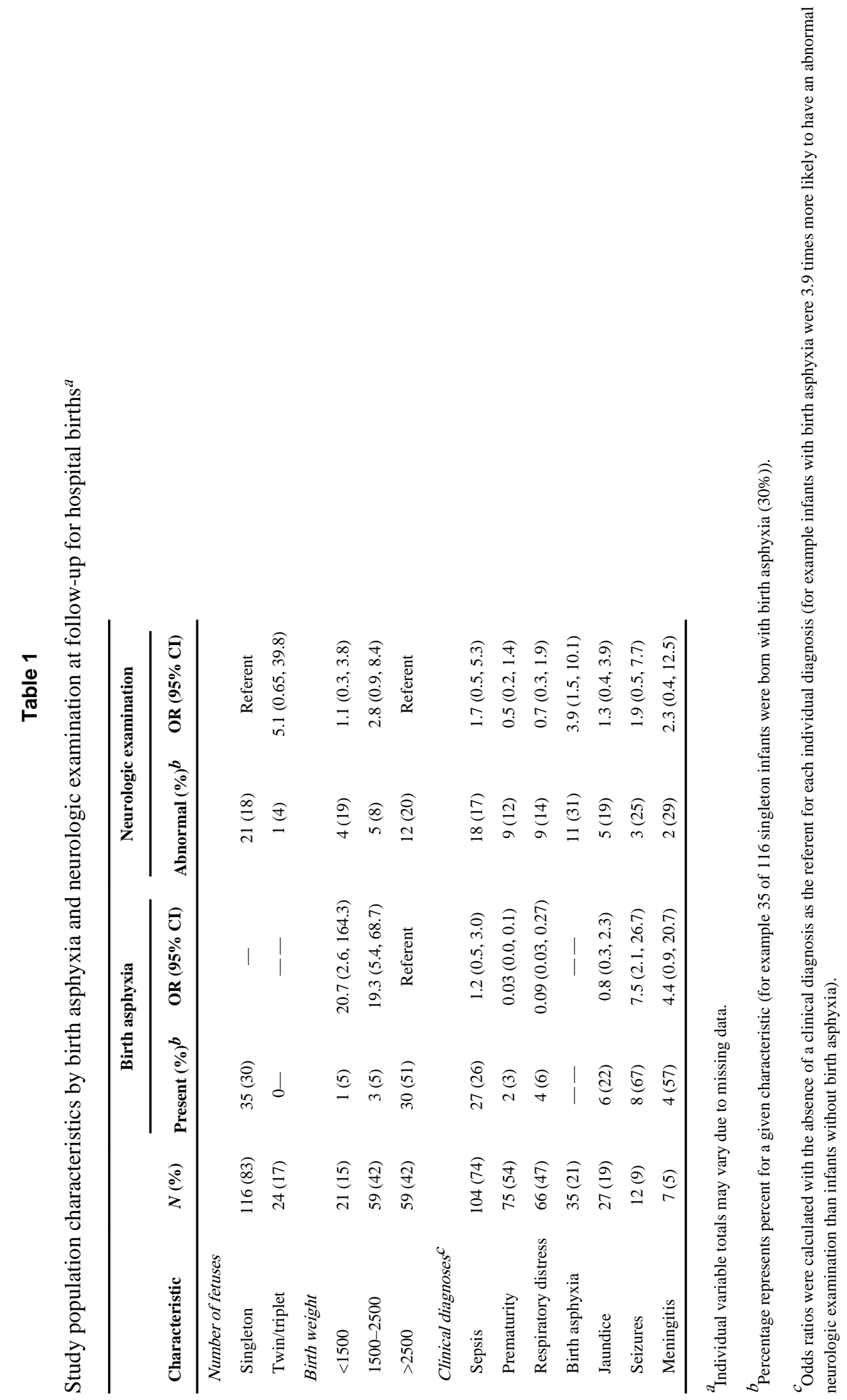




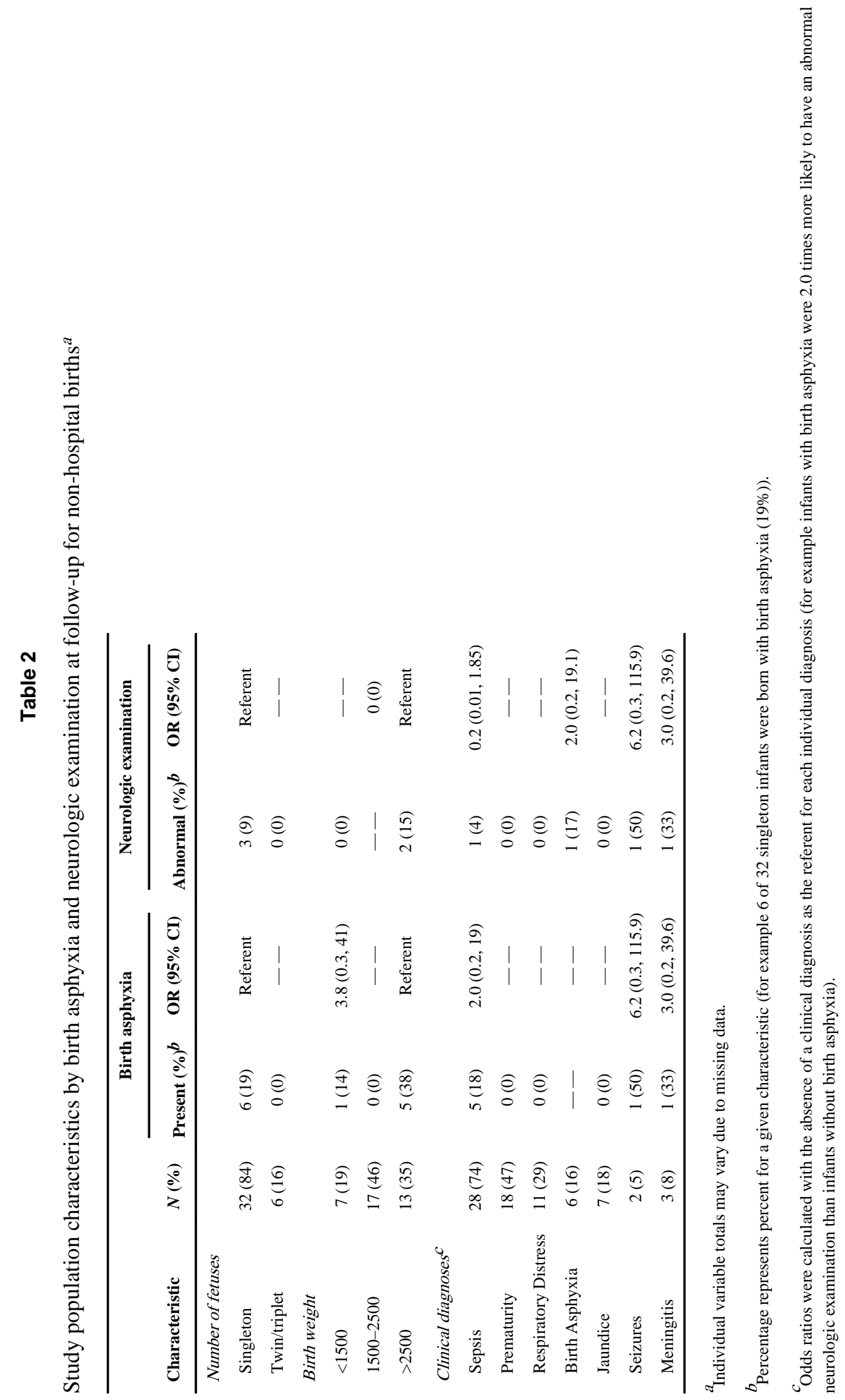

